\title{
Management of diabetes in patients on hemodialysis
}

\begin{abstract}
Abbreviations: HD, hemodialysis; ESRD, end stage renal disease; PPAR, peroxisome proliferator-activated receptor gamma; SU, sulphonylureas; GLP-1, glucagon like peptide-1; DPP-IV, dipeptidyl peptidase IV; GFR, glomerular filtration rate
\end{abstract}

\section{Introduction}

Managing diabetes in hemodialysis (HD) patients can be a daunting task. The various mechanisms at play in such patients may result in wide fluctuations in glucose levels. The interplay between uremia and HD employ antagonizing forces on insulin secretion, metabolism, action and tissue sensitivity, hence the wide variation in glycemic level. Management is further complicated by the absence of an ideal monitoring tool to evaluate glycemic control in diabetics on $\mathrm{HD}$. The use of HbA1c in uremic patients has been questioned in the last decade. ${ }^{1}$ Moreover, the target HbAlc in this population has not been established. ${ }^{2}$ Despite, diabetes being the most common cause of end stage renal disease in the United States, ${ }^{3}$ there is still controversy on how aggressive diabetes should be managed in HD patients. ${ }^{4-8}$

\section{To control or not to control}

A significant amount of evidence has shown higher HbAlc to be associated with a greater mortality rate in HD patients. ${ }^{9}$ On the other hand, aggressive glycemic control in this population has been discouraged due to an increased predisposition to hypoglycemic episodes seen in this population leading to increased mortality. ${ }^{9}$ Hence, maintaining adequate blood sugar level has to be a tight rope act but control of blood sugar should be a priority. A one size fit all approach should not be adopted and should be individualized.

\section{The ideal monitoring tool?}

$\mathrm{HbAlc}$ as a tool of monitoring glycemic control in end stage renal disease (ESRD) has disputed in recent years. ${ }^{1}$ In the presence of uremia hemoglobin gets carbamylated. This carbamylated hemoglobin is indistinguishable from glycated hemoglobin in commonly used charge based assays, hence values are falsely high. ${ }^{10-12}$ Moreover, HD patients are usually prescribed erythropoietin stimulating agents which result in reticulocytosis. Reticulocytes are slow to be glycated in comparison to mature red blood cells hence causing discrepancy in assessing true HbAlc level. Despite these limitations, in the absence of a better proven indicator using $\mathrm{HbAlc}$ is considered reasonable. Glycated albumin is considered to be better but awaits wide acceptability. ${ }^{12,13}$

\section{Mechanisms at play}

The wide variability in blood sugar level in a uremic setting is due to an inter-play of many factors. Uremia results in insulin resistance resulting in impaired glucose utilization which leads to a hyperglycemic state. Furthermore, the metabolic acidosis and decreased activated vitamin D-3 level seen in uremic patients suppresses insulin secretion which further worsens the hyperglycemia. ${ }^{14}$ On the other hand, 25\% of the insulin is metabolized in the kidney and with the decline in kidney function, the clearance of insulin decreases thus predisposing to hypoglycemia. Furthermore, the kidney also participates in gluconeogenesis hence once its function decline so does the blood

\author{
Volume 3 Issue 5 - 2016 \\ Muhammad Ahad Qayyum \\ Department of Nephrology, Bahria Town Hospital, Pakistan
}

Correspondence: Muhammad Ahad Qayyum, Department of Nephrology, Bahria Town Hospital, Lahore, Pakistan, Tel 0092 300-8432I50,Email drahadqayyum@gmail.com

Received: July 30, 2016 | Published: September 24, 2016

sugar level. Another factor is that patients on HD are exposed to dialysates which contain glucose hence can cause hyperglycemia. Which factor is more dominant than the other varies from one patient to the other, hence the recommendation of individualized care.

\section{Using oral hypoglycemic agents}

Majority of the oral hypoglycemic agents are not recommended for use in ESRD setting. The reason for this is multi-factorial but more often it is the bioaccumulation of the drug metabolites as majority are excreted by the kidney. ${ }^{15}$ Sulphonylureas (SU) work by increasing insulin secretion by stimulating the beta cells. They are strongly protein bound, hence are not dialyzable. ${ }^{16}$ All of the first generation SU i.e. chlorpropamide, tolbutamide, tolazamide are almost entirely excreted by the kidney. Since the kidney function in minimal in ESRD and the drug is not dialyzable, it can cause hypoglycemia thus are contraindicated in this setting. As far as second generation SU like glipizide, gliclazide and glimepiride are concerned, they are metabolized in the liver but most of their active metabolites are excreted by the kidney. ${ }^{17}$ The exception to this is glipizide that is metabolized in the liver to inactive-weakly active metabolites that are excreted by the kidney. Hence, glipizide is the only SU recommended for safe use in ESRD.

Biguanides like metformin are excreted via the kidney hence are bio-accumulated in renal failure predisposing to lactic acidosis. Biguanides are contraindicated in this setting. ${ }^{17}$ Thizolidinediones are peroxisome proliferator-activated receptor gamma (PPAR) agonists which enhance insulin sensitivity and reduce hepatic glucose production. ${ }^{18}$ They are not dialyzable as they are highly protein bound. They are almost entirely metabolized by the liver thus not accumulated in ESRD. However, these drugs have the potential to cause edema and precipitate heart failure as a result not considered ideal drugs to be used in HD patients. Alpha glucosidase inhibitors like acarbose decrease absorption of glucose from the intestine. These drugs are also accumulated in renal failure and are not recommended for use in this setting. Besides, the added disadvantage of gastrointestinal side effects has made this class loose favor. ${ }^{17}$

Meglitinides include repaglinide and nateglinide. They are new non sulphonylurea based insulin secretagogues. Nateglinide accumulates in renal failure and causes hypoglycemia. ${ }^{19}$ Repaglinide is metabolized in the liver and less than $10 \%$ is excreted via the kidney. Thus a reduced dose can be used in patients on HD to control 
blood sugar. Cost can be an issue. Glucagon like peptide-1 (GLP-1) agonists and dipeptidyl peptidase IV (DPP-IV) inhibitors stimulate glucose dependant insulin release, suppress postprandial glucagon release and slow gastric emptying. GLP-1 agonists like exenatide is not recommended in such patients. While DPP IV inhibitors like sitagliptin, saxagliptin and vildagliptin can be used in HD patients. ${ }^{20}$

\section{Insulin remains cornerstone of treatment}

Insulin clearance decreases with level of uremia as discussed earlier. Hence the dose of insulin should be halved once glomerular filtration rate (GFR) is less than $10 \mathrm{~mL} / \mathrm{min} .{ }^{21}$ Although a consensus approach is lacking but most authorities endorse using rapid acting insulin like lispro before each meal alongside close monitoring of blood glucose. ${ }^{17}$ Use of basal insulin has been controversial and reserved for those patients whose blood sugar is not under control with short acting insulin. Dose of basal insulin should similarly be halved at outset and then titrated up.

\section{Conclusion}

Education of the patient regarding diabetes is equally important with special emphasis on recognition and treating hypoglycemia. Most of the evidence discussed above is based on observational and nonrandomized studies as data is scarce on this topic. Insulin remains the cornerstone of treatment and if oral hypoglycemic drugs are to be used the available options includes glipizide (SU), sitagliptin, saxagliptin and repaglinide.

\section{Acknowledgments}

None.

\section{Conflicts of interest}

The author declares there is no conflict of interest.

\section{References}

1. Ansari A, Thomas S, Goldsmith D. Assessing glycemic control in patients with diabetes and end-stage renal failure. Am J Kidney Dis. 2003;41(3):523-531.

2. $2005 \mathrm{~K} / \mathrm{DOQI}$ clinical practice guidelines for cardiovascular disease in dialysis patients. Am J Kidney Dis. 4(Suppl 3):S1.

3. National Institute of Diabetes and Digestive and Kidney Diseases. United States Renal Data System: USRDS 2005 Annual Data Report. Bathesda, MD: National Institutes of Health. 2005.

4. Tzamaloukas AH, Murata GH, Zager PG, et al. The relationship between glycemic control and morbidity and mortality for diabetics on dialysis. ASAIOJ. 1993;39(4):880-885.

5. McMurray SD, Johnson G, Davis S, et al. Diabetes education and care management significantly improve patient outcomes in the dialysis unit. Am J Kidney Dis. 2002;40(3):566-575.
6. Oomichi T, Emoto M, Tabata T, et al. Impact of glycemic control on survival of diabetic patients on chronic regular hemodialysis: a 7-year observational study. Diabetes Care. 2006;29:1496-1500.

7. Williams ME, Lacson E, Teng M, et al. Hemodialyzed type I and type II diabetic patients in the US: Characteristics, glycemic control, and survival. Kidney Int. 2006;70:1503-1509.

8. Shurraw S, Majumdar SR, Thadhani R, et al. Glycemic control and the risk of death in 1,484 patients receiving maintenance hemodialysis. $\mathrm{Am}$ J Kidney Dis. 2010;55(5):875-884.

9. Williams ME, Lacson E, Wang W, et al. Glycemic control and extended hemodialysis survival in patients with diabetes mellitus: comparative results of traditional and time-dependent Cox model analyses. Clin J Am Soc Nephrol. 2010;5(9):1595-1601.

10. Scott MG, Hoffmann JW, Meltzer VN, et al. Effects of azotemia on results of the boronate-agarose affinity and ion-exchange methods for glycated hemoglobin. Clin Chem. 1984;30(6):896-898.

11. Wettre S, Lundberg M. Kinetics of glycosylated haemoglobin in uraemia determined on ion-exchange and affinity chromatography: no increase in the rate of glycosylation. Diabetes Res. 1986;3(2):107-110.

12. Freedman BI, Shihabi ZK, Andries L, et al. Relationship between assays of glycemia in diabetic subjects with advanced chronic kidney disease. Am J Nephrol. 2010;31(5):375-379.

13. Peacock TP, Shihabi ZK, Bleyer AJ, et al. Comparison of glycated albumin and hemoglobin A(1c) levels in diabetic subjects on hemodialysis. Kidney Int. 2008;73(9):1062-1068.

14. Mak RH. Intravenous 1,25-dihydroxycholecalciferol corrects glucose intolerance in hemodialysis patients. Kidney Int. 1992;41(4)1049-1054.

15. Charpentier G, Riveline JP, Varroud-Vial M. Management of drugs affecting blood glucose in diabetic patients with renal failure. Diabetes Metab. 2000;26(Supp1 4):73-85.

16. Skillman TG, Feldman JM. The pharmacology of sulfonylureas. Am J Med. 1981;70(2):361-372.

17. Snyder RW, Berns JS. Use of insulin and oral hypoglycemic medications in patients with diabetes mellitus and advanced kidney disease. Semin Dial. 2004;17(5):365-370.

18. Lubowsky ND, Siegel R, Pittas AG. Management of glycemia in patients with diabetes mellitus and CKD. Am J Kidney Dis. 2007;50(5):865-879.

19. Nagai T, Imamura M, Iizuka K, et al. Hypoglycemia due to nateglinide administration in diabetic patient with chronic renal failure. Diabetes Res Clin Pract. 2003;59(3):191-194.

20. Bergman AL, Cote L, Yi B, et al. Effect of renal insufficiency on the pharmacokinetics of sitagliptin, a dipeptidyl peptidase-4 inhibitor. Diabetes Care. 2007;30(7):1862-1864.

21. Mak RH, Defronzo RA. Glucose and insulin metabolism in uremia. Nephron. 1992;61(4):377-382. 